\title{
The Effect of the Red Light on Reactive Oxygen Species Production by Neutrophils in Vitro
}

\author{
H. NAwrocka-Bogusz* AND F. JAROSZYK \\ The Department of Biophysics, University of Medical Science, A. Fredry 10, 61-701 Poznań, Poland \\ The aim of this study was to investigate the effect of the red light $(630 \mathrm{~nm})$ upon reactive oxygen species \\ production by neutrophils in vitro. Blood samples were used for the study. The flow cytometry method was used \\ for estimation of hydrogen peroxide production. A statistically significant decrease of reactive oxygen species \\ production by neutrophils was observed. The level of the decrease varied and it was dependent on the kind of \\ spectrum of applied signals and different levels of energy light.
}

PACS: 87.50.W-, 87.17.-d, 82.30.Cf

\section{Introduction}

Neutrophils are highly specialized white blood cells, contributing to the immune response. Their basic function is the destruction of phagocytised microorganisms. One of intercellular destruction mechanisms of pathogens is the production of reactive oxygen species (ROS), called respiratory burst [1-3]. The mechanism of the respiratory burst consists in a more than ten times increase of the consumption of oxygen, as well as in the production and release of large quantities of superoxide radical anion $\left(\cdot \mathrm{O}_{2}^{-}\right)$outside the cell. The dismutation of this radical causes the formation of hydrogen peroxide $\left(\mathrm{H}_{2} \mathrm{O}_{2}\right)$. The respiratory burst causes the formation of large quantities of $\mathrm{O}_{2}^{-}$and $\mathrm{H}_{2} \mathrm{O}_{2}$ around the phagocyte cells. Hydrogen peroxide, as a reactively weak and electrically inert compound, can easily penetrate the cell's membrane and appear in several places of the cell completely different from those, in which it is being produced. Activated neutrophils are one of the main sources of ROS in the organism $[2,4]$.

The NADPH oxidase is an enzyme catalyzing the production of superoxide anion from oxygen and reduced nicotinamide adenine dinucleotide phosphate (NADPH). The NADPH oxidase is a key driving force in powering the microbicidal capacity of neutrophil cells. Mutations in any component of the NADPH oxidase prevent the effective functioning of the neutrophil. Normal functioning of neutrophils relies upon the concerted action between the NADPH oxidase and different ion channels, which enables neutrophils to prodigiously fulfill their important role when recruited to sites of inflammation or infection [5].

* corresponding author; e-mail: hnawrocka@ump.edu.pl
The electric qualities of the light, which is capable of generating electric excitation when absorbed, have to be taken into consideration. By definition, there is an intense oscillating electric field $(E)$ associated with visible and near infrared (NIR) photons. This field produces electrical current if free electrons are present in the medium. The presence of the current leads to the absorption of the energy and conversion into heat (the Joule heating). When the electric field of a photon that oscillates at the same electronic resonant frequency approaches the electron, it is absorbed and electronic excitation occurs. Any other frequencies are not absorbed, but they modify the frequency of oscillation of bound electrons. Because of this interaction, the light slows in the medium and is refracted, and some of its energy is stored in the medium in the form of electric potential energy. This exchange of energy produces electric dipole transitions of the material, i.e. dipoles change their internal characteristics due to a displacement of bound charges [6].

When cells are irradiated with visible wavelengths a variety of stimulatory effects are observed in their metabolism. The first goal of irradiation with light of $630 \mathrm{~nm}$ wavelength are mitochondrions. The light-induced electric field is able to change the membrane potentials in the mitochondrial membrane by transferring charges from the outer side to the inner side or directly by a polarization of membrane dipoles [6]. Research on the influence of monochromatic visible and near-infrared light showed that the use of light of $633 \mathrm{~nm}$ length causes an increase in mitochondrial membrane potential $(\Delta \Psi)$ and proton gradient $(\Delta \mathrm{pH})$, and also modifies the NADH dehydrogenase reaction and increases the rate of ADP/ ATP. NADH dehydrogenase is considered to be a probable photoacceptor of the red light. Biological cell response to the visible light irradiation is caused by phys- 
ical and/or chemical changes of photoacceptor, components of respiratory chain and cytochrome c oxidase [7].

A consequence of the absorption of light quanta is also the depolarization of the cell membrane and calcium ions $\left(\mathrm{Ca}^{2+}\right)$ influx into the cell [7].

Cells with less than normal intracellular $\mathrm{pH}$, that is with the "redox" state shifted in the direction of reduction, are more sensitive to the stimulating light in comparison with the cells the individual parameters of which were optimal or close to optimal [7].

Other studies showed that a low-level laser therapy decreased the production of ROS by neutrophils, the levels of neutrophil anti-apoptotic factors, the IL- $1 \beta$ concentration, and lung myeloperoxidase activity after lipopolysaccharide-induced lung inflammation [8].

The red light is used singly as a physiotherapy method [1].

We have investigated whether different density of the red light energy and different signal spectrum can cause effect on reactive oxygen species production by neutrophils.

\section{Material and methods}

\subsection{Material}

Blood from healthy volunteers was used for the purpose of the study. Lithium heparin was used as the anticoagulant.

\subsection{Methods}

DCFH-DA (Sigma-Aldrich, USA) was used to show the presence of hydrogen peroxide [3]. For one measurement the blood from one person was divided into 4 test tubes containing $50 \mu \mathrm{l}$ of blood each. $10 \mu \mathrm{l}$ of DCFH-DA diluted solution was added to each test tube. Two of the examined samples were irradiated by the red light of wavelength $630 \mathrm{~nm}$ generated by a special generator - Viofor JPS for $30 \mathrm{~min}$ at room temperature. The other test tubes were kept as a control in dark, in room temperature. The respiratory burst was induced with PMA (phorbol 12-myristate, 13-acetate; Sigma-Aldrich, USA), to produce submaximal stimulation of the respiratory burst. After the application of the red light $(\mathrm{R})$, $2 \mu \mathrm{l}$ of PMA solution was added to one of the control test tubes and one of the influenced by R. After a 15 min incubation at room temperature in the dark, $700 \mu \mathrm{l}$ of red blood cells lysis solution was added to each test tube. After a 15 min test tubes incubation at room temperature in the dark, the flow cytometry measurement was made. The efficiency of respiratory burst (the production of $\mathrm{H}_{2} \mathrm{O}_{2}$ ) in individual cells was estimated via the flow cytometry by using the intracellular oxidative transformation of DCFH-DA $\left(2^{\prime} 7^{\prime}\right.$-dichlorofluorescin diacetate) to the fluorescent DCF $\left(2^{\prime} 7^{\prime}\right.$-dichlorofluorescin $)$ $\left(\lambda_{\text {excitation }}=498 \mathrm{~nm}, \lambda_{\text {emission }}=522 \mathrm{~nm}\right)[3,9,10]$. The intensity of DCF fluorescence refers to the quantity of produced hydrogen peroxide. Cytofluorimeter FACScan
Becton Dickinson equipped with $15 \mathrm{~mW}$ argon laser was used in case of this research.

The frequencies of red light base impulses are in the range $(180 \div 195) \mathrm{Hz}$, the frequencies of impulses pack are $(2.8 \div 7.6) \mathrm{Hz}$, the frequencies of pack groups are $(12.5 \div$ $29) \mathrm{Hz}$, and series $(0.08 \div 0.3) \mathrm{Hz}$. The form of impulses is close to the peak shaped. The shapes of impulses, their frequencies and time dependence relations are described by programs called P2, P3. In these programs the forms of impulses are similar, but their spectrum is different $[11,12]$.

We used two kinds of applications methods: M1 and M2 according the Viofor JPS device. M1 means an application with the constant intensity during the whole application time. M2 means an application with rising intensity to the chosen value. After reaching the chosen intensity, the value of impulse strength decreases. This process is repeated periodically.

We used combinations of programs and ways of application, we referred to them in general as "programs": M1P2, M1P3, M2P2, and M2P3. We used three different levels of energy light for each of the "programs" marked: $3,5,10$ on the Viofor device. Those numbers $(3,5,10)$ refer to the values of densities of the red light energy for P2 equalled: $1.12,1.17$ and $1.19 \mathrm{~J} / \mathrm{cm}^{2}$. The values of densities of the red light energy for P3 were: 1.17, 1.20 and $1.23 \mathrm{~J} / \mathrm{cm}^{2}$.

For statistical analysis, Wilcoxon test and descriptive statistics were used.

\section{Results}

In our work, we chose the following abbreviations for the samples:

1 - a sample of blood unstimulated with PMA, without the influence of the red light $(\mathrm{R})$;

2 - a sample of blood stimulated with PMA, without the influence of the red light;

3 - a sample of blood unstimulated with PMA, under the influence of the red light;

4 - a sample of blood stimulated with PMA, under the influence of the red light;

(4-3) - respiratory burst in samples influenced by the red light;

(2-1) - respiratory burst in native samples.

We compared sample 1 with sample 3 , sample 2 with sample 4 and subtraction of sample 3 from sample 4 (4-3) with subtraction of sample 1 from sample $2(2-1)$.

A statistically significant decrease of reactive oxygen species production by neutrophils was observed during the experiments (Figs. 1, 2).

Programs: M2P3, M1P3, M1P2 caused similar statistically significant $(p \leq 0.02)$ decrease of DCF fluorescence of unstimulated neutrophils when three different light densities were applied (Fig. 1a). The smallest red light effect was observable when density of energy equalled 1.17 or $1.20 \mathrm{~J} / \mathrm{cm}^{2}$ for all abovementioned programs $(23-25 \%$ ROS decreasing in samples under the influence of $\mathrm{R}$ in 


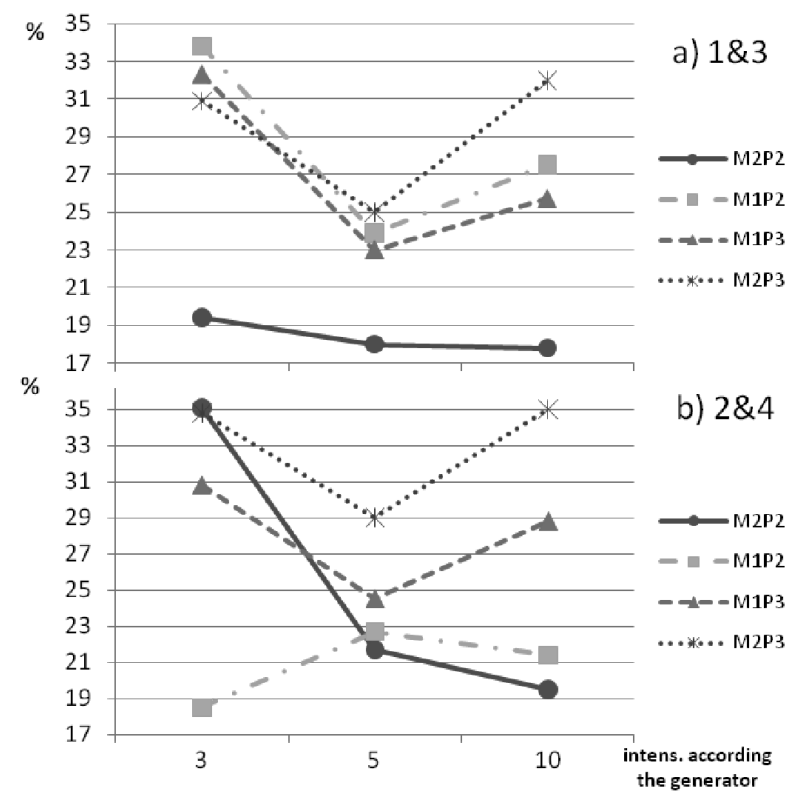

Fig. 1. Percentage of DCF fluorescence decrease of (a) PMA unstimulated neutrophils, (b) PMA stimulated neutrophils.

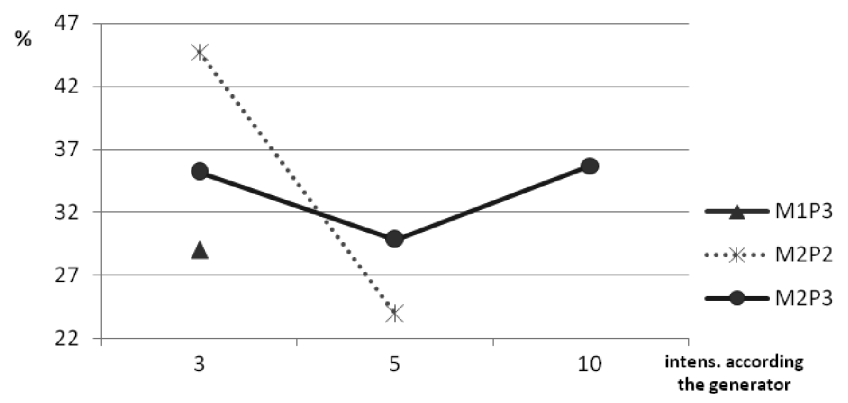

Fig. 2. Quantity of the respiratory burst.

comparison with those without the influence of this factor). M2P2 program influenced $(p \leq 0.02)$ the unstimulated neutrophils to the least extent, in comparison to the other programs, the decrease level of DCF fluorescence was in the range 18-20\% for all used light energy densities. The highest changes were caused by programs M2P3 or M1P3 or M1P2, when the generator was adjusted to the lowest used energy density. Only the highest energy density, when the program M2P3 was used, caused almost the same decrease of DCF fluorescence (32\%) that the lowest density of this program (31\%) (Fig. 1a).

The biggest statistically significant $(p \leq 0.01)$ changes of DCF fluorescence of PMA stimulated neutrophils was caused by M2P3 program (Fig. 1b). The level of decrease was $35 \%, 29 \%$, and $35 \%$ respectively for the red light energy densities: $1.17,1.20$, and $1.23 \mathrm{~J} / \mathrm{cm}^{2}$. For the same energy densities but with M1P3 program using, the red light caused decrease of ROS production $(p=0.01)$ respectively by $31 \%, 25 \%, 29 \%$. M2P2 pro- gram also caused large decrease of DCF fluorescence $(35 \%, p=0.01)$ when energy density was $1.12 \mathrm{~J} / \mathrm{cm}^{2}$ in comparison to those samples without the influence of the red light. The energy density: 1.17 and $1.19 \mathrm{~J} / \mathrm{cm}^{2}$ for this program caused $21.5 \%$ and $19.5 \%$ decrease of DCF fluorescence. We observed inversed relation with the decrease $(p=0.02)$ of ROS production by the use of the M1P2 program. The smallest energy density caused the lowest decrease (18\%) of DCF fluorescence. Higher level of energy density entailed the greatest changes (22.5\%) after application of this program and the biggest used energy density caused smaller DCF fluorescence decrease $(21.5 \%)$ than lower energy density of red light (Fig. 1b).

We estimated the value of the quantity of the respiratory burst, in comparison to the respiratory burst in native samples $(2-1)$ and the respiratory burst in the samples influenced by red light $(4-3)$. We obtained statistically significant changes $(p \leq 0.02)$ of quantity of the respiratory burst when the M1P3, M2P2, M2P3 programs were applied (Fig. 2). The respiratory burst in the samples influenced by $\mathrm{R}$ was decreased in comparison to the respiratory burst in native samples. The M1P3 caused $28 \%$ of the DCF fluorescence decrease after the red light irradiation only for $1.17 \mathrm{~J} / \mathrm{cm}^{2}$ of energy light density. The M2P2 program caused a decrease of respiratory burst by $45 \%$ and $24 \%$ when the energy density was, respectively, 1.12 , and $1.17 \mathrm{~J} / \mathrm{cm}^{2}$. The M2P3 caused changes: $35 \%, 30 \%$ and $36 \%$ for all used energy densities.

\section{Discussion}

The red light can influence a few cellular mechanisms of ROS production by neutrophils, such as the mechanism of PKC activation, intra- and extracellular $\mathrm{Ca}^{2+}$ influx or electron current generated by NADPH oxidase.

Protein kinase $\mathrm{C}(\mathrm{PKC})$ isoforms were implicated in the oxidative burst as potential key activators of NADPH oxidase, because the stimulation of neutrophils with phorbol esters activates PKC and leads to a strong oxidative response [13]. PKC isoforms are required for phosphorylation of $\mathrm{p} 47$ phox (cytosolic subunits of NADPH oxidase), resulting in the assembly and activation of phagocytic NADPH oxidase [13].

Intra- and extracellular $\mathrm{Ca}^{2+}$ are required for full activation of the respiratory burst of human neutrophils, and the $\mathrm{Ca}^{2+}$ influx from the extracellular space plays an important role either in the generation of reactive oxygen metabolites or in the activation of PKC [14].

When cells are irradiated with red and NIR light, oscillations in calcium concentration are induced by releasing $\mathrm{Ca}^{2+}$ stored in the endoplasmic reticulum [6].

Specific neutrophil responses can be stimulated by the potent chemo-attractants. Binding of chemo-attractants to receptors on the neutrophil plasma membrane releases intracellular calcium $\left(\mathrm{Ca}^{2+}\right)$ via the phospholipaseinositol triphosphate pathway. This depletion of $\mathrm{Ca}^{2+}$ in turn leads to $\mathrm{Ca}^{2+}$ influx via store operated channels 
resulting in elevated $\mathrm{Ca}^{2+}$ levels that are thought to be implicated in several $\mathrm{Ca}^{2+}$ dependent pro-inflammatory responses. In case of neutrophils, refilling of these stores via store operated channels (a phenomenon known as capacitative $\mathrm{Ca}^{2+}$ entry) is mediated by the NADPH oxidase. An important feature of the neutrophil NADPH oxidase is that it generates an electron current which depolarises the membrane. The electrogenic characteristic of the NADPH oxidase means that its inhibition will also abrogate the membrane depolarisation and augment $\mathrm{Ca}^{2+}$ entry into the cell. The depolarisation thereby alters the electrochemical gradient across the neutrophil plasma membrane and limits $\mathrm{Ca}^{2+}$ entry into the cell. The NADPH oxidase puts a protective restraint on $\mathrm{Ca}^{2+}$ entering into the neutrophil [5].

The effects of a light-induced electric field will be nonspecific because its intrinsic mechanism is to displace any bound charge in a wavelength dependent manner or to create membrane potentials. This does not mean that any enzyme or chemical bond will be functionally affected by the induced electric field. The effect will depend on the level of cellular concentration of substrates, products and enzymes. These levels of concentration will be determined by the biochemical pathways that are active in the cell when irradiation occurs. Therefore, they will depend on the metabolic state of the cell. An increase in $\mathrm{Ca}^{2+}$ concentration will initiate several metabolic processes. Enzymatic activity will be increased due to both the effects of $\mathrm{Ca}^{2+}$ and the activation of enzymatic molecules by the light. The metabolism will be powered by the direct action of light on mitochondria, the effects of $\mathrm{Ca}^{2+}$ on mitochondria, and the excitation of ATP in cytoplasm by the irradiation [6].

The system of antioxidant enzymes which can be activated by the red light is: superoxide dismutase (SOD), catalase (CAT) and glutathione peroxidase (GSH-Px). Those enzymes reduce reactive oxygen species $\left(\mathrm{H}_{2} \mathrm{O}_{2}\right.$ is reduced in the presence of catalase and glutathione reductase to water and molecular oxygen) [15]. If they are activated, the level of ROS is reduced, as we observed in our research.

\section{Conclusions}

The red light influenced the activity of neutrophils causing a decrease of $\mathrm{H}_{2} \mathrm{O}_{2}$ production. A statistically significant decrease of DCF fluorescence was observed. M1P3 and M2P3 program caused similar results in both stimulated or unstimulated neutrophils for all used light energy densities. M1P2 program decreased DCF fluorescence of stimulated or unstimulated neutrophils at the same level, only when the intensity was $1.17 \mathrm{~J} / \mathrm{cm}^{2}$. For
M2P2 program it was observable that the higher density of the energy of light the lower decrease of $\mathrm{H}_{2} \mathrm{O}_{2}$ production both by stimulated and unstimulated neutrophils. Each of the programs used by us can be useful in treatment of many diseases [8], the result depending on the achieved level of ROS reductions.

A variety of autoimmune and inflammatory diseases are mediated in some part by neutrophils, for example: gout, rheumatoid arthritis, pleurisy, peritonitis, and appendicitis. A variety of airway diseases are mediated by neutrophil infiltration: chronic obstructive pulmonary disease, bronchiectasis, bronchiolitis, cystic fibrosis, and certain forms of asthma. In such cases of chronic brooding diseases, home light therapy could be also used for potential decrease of exacerbations and hospital admissions and for improvement of wound healing [8].

\section{References}

[1] H. Nawrocka, B. Poniedziałek, F. Jaroszyk, K. Witorowicz, Polish J. Environ. Stud. 15, 28 (2006).

[2] C. Dahlgren, A. Karlsson, J. Immunol. Methods 232 , 3 (1999).

[3] A. Gomes, E. Fernandes, J. Lima, J. Biochem. Biophys. Methods 65, 45 (2005).

[4] A. Mayer-Scholl, P. Averhoff, A. Zychlinsky, Curr. Opin. Microbiol. 7, 62 (2004).

[5] M.D. Salmon, J. Ahluwalia, Biochem. Biophys. Res. Commun. 384, 87(2009).

[6] A. Amat, J. Rigau, R.W. Waynant, I.K. Ilev, J.J. Anders, J. Photochem. Photobiol. B 82, 152 (2006).

[7] T. Karu, J. Photochem. Photobiol. B 49, 1 (1999).

[8] M.C. Morgan, R.M. Rashid, Int. Immunopharmacol. 9, 383 (2009).

[9] W. Jakubowski, G. Bartosz, Cell. Biol. Int. 24, 757 (2000).

[10] S. Walrand, S. Valeix, C. Rodriguez, P. Ligot, J. Chassagne, M. Vasson, Clin. Chim. Acta 331, 103 (2003).

[11] W. Deka, Biuletyn informacyjny Med\&Life Polska 6 , 15 (2001), (in Polish).

[12] W. Deka, Szkolenie o produkcie, Med\&Life Polska, Warszawa 2004 (in Polish).

[13] A. Bertram, K. Ley, Arch. Immunol. Ther. Exp. 59, 79 (2011).

[14] L. Bei, T. Hu, Z. Ming, X. Qian, Shen, Biochim. Biophys. Acta 1404, 475 (1998).

[15] G. Cieślar, J. Zalejska-Fiolka, E. Birkner, S. Kasperczyk, A. Sieroń, Phys. Med. 20, Supp. 1, 25 (2004). 